\title{
Alpha-Blockers for the treatment of chronic prostatitis in combination with antibiotics Stellungnahme zur Arbeit Barbalias et al. (1998) J Urol 159: 883-887
}

E. ine funktionell gestörte Blasenentleerung bei Patienten mit "Prostatitis“ wird seit 2 Jahrzehnten diskutiert $[1,6$, $7,13]$. Wir selbst haben in einem großen Patientengut von 235 Männern mit gesicherter chronischer Prostatitis bei $33 \%$ der Fälle eine funktionelle Blasenentleerungsstörung gesichert [15]. Andererseits muß darauf hingewiesen werden, daß eine urodynamisch gesicherte, „echte" Obstruktion mit einer Prävalenz von $1,6 \%$ in diesem Patientengut deutlich geringer ist als bisher vermutet [10].

Nun ist die symptomatische Besserung das vorrangige Ziel der Prostatitistherapie. Dies kommt auch in der neuen Klassifikation der NIH zum ProstatitisSyndrom zum Ausdruck [16]. Barbalias hat bereits 1990 Schmerzsymptome bei funktioneller Blasenentleerungsstörung durch einen erhöhten urethralen Verschlußdruck mit konsekutivem intraprostatischen Reflux erklärt und für Patienten mit „Prostatodynie“ den Begriff „Painful Male Urethral Syndrome“ vorgeschlagen [2]. Daß ein gestörter laminarer Urinfluss in der prostatischen Harnröhre sich auf die Ätiopathogenese auswirkt, ist eine These, die seit Jahren unter dem Schlagwort „Prostatischer Reflux"diskutiert wird [4,9]. Re- und Influx von sterilem Urin [6, 8], Mikroorganismen [4], Spermatozoen [11] und Uratund Kreatinin-Metaboliten [14] sind als Auslöser einer Entzündung und anhaltender Symptomatik diskutiert worden. Dabei ist die Behandlung mit $\alpha$-Blokkern seit Jahren ein Standard-Therapievorschlag bei allen Formen eines Prostatitissyndroms. Neu ist der Versuch, die Besserung der Symptomatik zu validieren $[5,12]$ und jetzt auch bei chronisch- bakterieller Prostatitis durch gemeinsame Gabe eines Antibiotikums und eines alpha-Blockers die schlechte bakteriologische Ansprechrate bei derartigen Patienten zu verbessern [3]. Ohne jetzt „beckmesserisch" einzelne, methodisch unzulängliche Punkte der Studie überzubewerten, denke ich, daß die Anwendung von alpha-Blockern bei Patienten mit Prostatitis ein pathogenetisch sinnvolles und symptomatisch wirksames Prinzip darstellt.

\section{Literatur}

1. Barbalias GA, Meares EM, Sant GR (1983) Prostatodynia: clinical and urodynamic characteristics. J Urol 130: 514-517

2. Barbalias GA (1990) Prostatodynia or painful male urethral syndrome. Urology 36: 146-153

3. Barbalias GA, Nikiforidis G, Latsikos EN (1998) Alpha-Blockers for the treatment of chronic prostatitis in combination with antibiotics. J Urol 159: 883-887

4. Blacklock NJ (1991) The anatomy of the prostate: relationship with prostatic infection. Infection (Suppl 3) 19: 111-114

5. de la Rosette JJMCH, Karthaus HFM, van Kerrebroeck PhEVA, de Boo Th, Debruyne FMJ (1992) Research in "Prostatitis syndromes": the use of alfuzosin in patients mainly presenting with micturition complaints of an irritative nature and confirmed urodynamic abnormalities. Eur Urol 22: 222-227

6. Hellstrom WJG, Schmidt RA, Lue TF, Tanagho EA (1987) Neuromuscular dysfunction in nonbacterial prostatitis. Urology 30: 183-188

7. Kaplan StA, et al. (1997) Pseudodyssynergia (contraction of the external sphincter during voiding) misdiagnosed as chronic nonbacterial prostatitis and the role of biofeedback as a therapeutic option. J Urol 2234-2237
8. Kirby RS, Lowe D, Bultetude MJ, Shuttleworth KED (1982) Intraprostatic urinary reflux: an etiological factor in abacterial prostatitis. Br J Urol 54: 729-731

9. Madersbacher $\mathrm{H}$ (1983) Blasenentleerungsstörung und chronische Prostatovesikulitis. In: Brunner H, Krause W, Rothauge CF, Weidner W (Hrsg) Chronische Prostatitis. Schattauer, Stuttgart, S319-327

10. Mayo ME, Ross SO, Krieger JN (1998) Few patients with "chronic prostatitis" have significant bladder outlet obstruction. Urology 52: 417-421

11. McClinton S, Eremin O, Miller JD (1990) Inflammatory infiltrate in prostatic hyperplasia - evidence of a host response to intraprostatic spermatozoa? $\mathrm{Br} J$ Urol 55: 606-610

12. Neal DE, Moon TD (1993) Use of terazosin in prostatodynia and validation of a symptom score questionnaire. Urology 43: 460-465

13. Palmtag H, Riedasch $G$ (1983) Medikamentöse Behandlung funktioneller Blasenentleerungsstörungen bei Prostatitis. In: Brunner H, Krause W, Rothauge CF, Weidner W (Hrsg) Chronische Prostatitis. Schattauer, Stuttgart, S347-354

14. Persson B-E, Ronquist G, Ekblom M (1996) Ameliorative effect of allopurinol on nonbacterial prostatitis: a parallel doubleblind controlled study. J Urol 155: 961-964

15. Weidner W, Ludwig M (1994) Diagnostic management in chronic prostatitis. In: Weidner W, Madsen PO, Schiefer HG (Hrsg) Prostatitis. Etiopathology, Diagnosis and Therapy. Springer, Berlin Heidelberg New York, S49-65

16. Weidner W (1999) Eine neue ProstatitisKlassifikation. Urologe [A] 38: 185

Prof. Dr. W. Weidner

Urologische Klinik der Justus-Liebig-Universität, Klinikstraße 29, D-35392 Gießen 